\title{
Evolución cronológica del trasplante renal en España
}

\author{
Pérez Albacete M. \\ Hospital Universitario Virgen de la Arrixaca. Murcia. \\ Actas Urol Esp. 2006;30(8):735-748
}

\section{RESUMEN}

EVOLUCIÓN CRONOLÓGICA DEL TRASPLANTE RENAL EN ESPAÑA

Introducción: Pretendemos establecer la evolución cronológica que ha llevado el trasplante renal en España. Apoyados en la heurística recogemos desde las primeras experiencias y trabajos científicos publicados a comienzos de los años sesenta hasta los de finales del siglo XX en el que el procedimiento se encuentra ya consolidado.

Material y método: Revisamos la bibliografia urológica española, las publicaciones de trasplante renal, las de diálisis y las nefrológicas y cuantas referencias bibliográficas hemos localizado además de aportar nuestra experiencia personal.

Resultados: El afán de ayudar a los pacientes en insuficiencia renal terminal fue el motor que impulsó a los urólogos españoles a comenzar con la práctica de los trasplantes renales. En mayo de 1960 el Dr. Martínez-Piñeiro efectúa el primero, sin resultado satisfactorio y es en julio de 1965 cuando el equipo del Dr. Gil-Vernet realiza un trasplante de cadáver con larga sobrevida del paciente y del órgano. Con los avances en los tratamientos inmunosupresores, el procedimiento se consolida como la mejor opción para el tratamiento de la insuficiencia renal y se suman a los trasplantes los principales hospitales del país.

Conclusiones: Los urólogos españoles, con su esfuerzo, dedicación y confianza en los resultados de la técnica, son los iniciadores y principales responsables de la actividad quirúrgica del implante y únicamente cuando el beneficio era evidente se movió la Administración a establecer las normas para su desarrollo; tras la constitución de la ONT, con su despliegue de medios, se logra que España alcance y afiance una primacía mundial tanto en número de donantes como de implantes.

Palabras Clave: Historia del trasplante renal. Trasplante renal en España. Trasplantación renal.

\section{ABSTRACT}

\section{CHRONOLOGICAL EVOLUTION OF THE RENAL TRANSPLANT IN SPAIN}

Introduction: We try to establish the chronological evolution that has taken the renal transplant in Spain. Supported on the heuristic one we gather from the first experiences and scientific works published at the beginning of the sixties up to those of the finals of the XXth century in which the procedure is already consolidated.

Material and method: We check the Spanish bibliography urology, the publications of renal transplant, those of dialysis and the nefrology and all the bibliographical references we have located beside contributing our personal experience.

Results: The zeal to help the patients in renal terminal insufficiency was the engine that stimulated the Spanish urologist to begin with the practice of the renal transplants. In May, 1960 the Dr. Martinez-Piñeiro carries out the first one, without satisfactory result and is in july, 1965 when the team of the Dr. Gil-Vernet realizes a transplant of corpse with long billiard cue sobrevida of the patient and of the organ. With the advances in the treatments immunosuppressants, the procedure is consolidated as the best option for the renal insufficiency treatment and the Spain principal hospitals add to the transplants.

Conclusions: The Spanish urologist, with their effort, dedication and confidence in the results of the technology, are the beginning and principal persons in charge of the surgical activity of the implant and only when the benefit was evident the Administration moved to establishing the procedure for its development; after the constitution of the ONT, with its unfolding of means, they achieves that Spain reaches and guarantees a world primacy so much in number of donors as of implants.

Keywords: History of the renal transplant. Renal transplant in Spain. Renal trasplantatión. 
Hasta los años sesenta del siglo pasado los pacientes con insuficiencia renal aguda se veían abocados a una muerte cierta, la diálisis y el riñón artificial aún no se encontraban introducidos en el arsenal terapéutico ordinario y es a partir de esa fecha cuando se comienzan a aplicar a estos enfermos. La primera hemodiálisis extracorpórea realizada en España la hizo el Dr. Rotellar, el 25 de febrero de 1957, en el Hospital de la Cruz Roja de Barcelona, para lo que utilizó un riñón artificial del modelo diseñado por el Dr. Kolff, en Holanda, en 1943, modificado y construido por él, de los que, en ese momento, únicamente había otros seis en Europa, en Kampen (Holanda), Leeds (Inglaterra), Lund (Suecia), París y Génova ${ }^{1}$; en esa época no existía aún la especialidad de Nefrología y éramos los urólogos los responsables de atender a estos enfermos en fase terminal de su proceso renal de ahí que en la Reunión Nacional de Urología (XXVI Congreso), celebrada en Valencia en mayo de 1961, los Drs. Tramoyeres y Lorca García presentaran una comunicación de su experiencia en el Hospital de la Cruz Roja de esa ciudad con un aparato dializador del modelo Kolff-Merrith, "Fallo renal agudo por intoxicación barbitúrico aguda, recuperado por aplicación de riñón artificial»² (Fig. 1).

El Dr. D. Carlos Jiménez Díaz (1898-1967), director del Instituto de Investigaciones Clínicas y Médicas en la Clínica de la Concepción de Madrid, dado su gran interés en estimular cual- quier avance de la Medicina potenció la experimentación en el trasplante animal ${ }^{3}$ y propuso, en 1958, algunas líneas de investigación, que fueron iniciadas por el Dr. Párraga Lázaro quien, en 1962, publicó ya un estudio en los Anales de la Academia de Ciencias de Nueva York ${ }^{4}$, en el que demostraba el valor inmunosupresor de la 6-mercaptopurina, preparado con el que un año antes el profesor Roy Calne había observado la supervivencia de los perros trasplantados a los que se les había suministrado la droga ${ }^{5}$, trabajos que, continuados, dieron lugar a la tesis doctoral del Dr. Vela Navarrete, en 1965, Mecanismos del rechazo del homoinjerto renal ${ }^{6}$ (Fig. 2).

El Dr. D. José Antonio Martínez-Piñeiro llevaba varios años interesado en los trasplantes, había realizado prácticas de autotrasplante en perros en el Instituto Cajal bajo la dirección de los Drs. D. Alfonso de la Peña y D. Fernando Castro seguidas por trabajos anatómicos de disección en el cadáver con la finalidad de familiarizarse con la vía de abordaje y de conocer los diversos tipos de pedículo vascular renal y de los vasos ilíacos; ante un paciente monorreno, con una tuberculosis renal de evolución progresiva e irreversible de la enfermedad, como única solución para resolver su situación se plantea, al tener un hermano gemelo homocigótico, efectuar un trasplante renal, que hasta el momento eran los únicos que habían tenido una buena evolución y lo efectúa el 24 de mayo de 1960 en el Hospital Provincial de Madrid; fue el primero realizado en España, la operación no presentó dificultad técnica pero no se logra la función del riñón debido al fallecimiento del paciente a los cuatro días, este caso lo presenta en la Reunión Anual de la AEU, en mayo de 1961, y publica en la Revista Clínica Española 7 , el 31 de julio de 1961: presentó un colapso vascular periférico que tuvo como causa primaria un hematoma retroperitoneal, el cual dio lugar a una infiltración de mesos y parálisis intestinal (Fig. 3). El segundo implante renal de donante vivo tiene lugar el 7 de

FIGURA 1. Reunión anual de la AEU, Valencia, 1961. 


\section{O R I G I N A L E S}

MECANISMO DE RECHAZO DEL HOMOINJERTO RENAL $\left(^{*}\right)$

$$
\begin{aligned}
& \text { R. Veta Navarkete } \\
& \text { Fundación Jiménez diaz } \\
& \text { INTroducción }
\end{aligned}
$$

El rechazo de un homoinjerto renal se debe a un proceso de indudable estirpe inmunológi${ }^{02},{ }^{214},{ }^{21 b},{ }^{71},{ }^{\text {2ha }}$. Esta afirmación relativa a los trasplantes renales, ha sido facilitada por las innumerables experiencias, realizadas previamente, con injertos tumorales y de piel ${ }^{2},{ }^{3}, ?, 11 \mathrm{n}$,

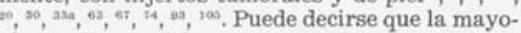
ría de ellas se deben al equipo de MrDawar, Bilitngham, Brent, Sparrow, Grbson, Gorer, etcétera. Aunque las observaciones fundamentales no hayan sido originariamente suyas, su confirmación y ampliación constituyeron el motivo de sus clásicas experiencias $,{ }^{3},{ }^{12},{ }^{12},{ }^{13},{ }^{30},{ }^{70},{ }^{23},{ }^{43}$.

FIGURA 2. Tesis doctoral, Dr. Vela Navarrete.

to. Esta condición se cumple entre gemelos univitelinos y entre animales altamente cruzados entre si, siguiendo el sistema hermano-hermana ${ }^{s i a-b}$. También es virtualmente satisfecha, cuando el receptor es el híbrido F1 de dos de tales razas y son sus padres los donantes, con tal que sean del mismo sexo o femenino el donante y el receptor masculino (EICHWABD, SIILMSER y WHEELER, 1955 y 1957)

4. Se comprueba por Michunson y otros 4. Se comprueba por Mrchunson y otros
(1953) ${ }^{\text {at }}$, cs, tas, que la sensibilidad producida por un trasplante de piel se puede transmitir, a otro animal de la misma cepa, mediante suspensiones de cŚlulas linfáticas y no por sangre total o suero, incluso tratándose de sueros hiperinmunes. Transmisión pasiva.

5. Se descubre, en animales que han rechazado un homoinjerto, la presencia de anticuerpos hemaglutinantes y citotóxicos (GoRER, 1957; GoRER y AMOS, 1954; CoOMBs, 1956; etc.).

6. BILlingham, Brent y MEdawar demuestran la llamada tolerancia inmunológica natural, describen sus cualidades y consiguen producirla artificialmente, en ratas (1954)? Estas

\section{$\longrightarrow$ $+2$ \\ TRASPLANTACION RENAL ENTRE GEMELOS HOMOZIGOTICOS \\ J. A. Martínez-Piñeiro. \\ Jefe Clínico del Servicio de Urologia del Hospital Provincial
de Madrid. Prof. Jefe: Dr. Pérez Castro.}

\section{FIGURA 3. Primer trasplante renal. Dr. M. Piñero.}

febrero de 1961, en la clínica madrileña Ntra. Sra. de la Paloma, por el Dr. D. Carlos Younger de la Peña, de un padre a su hijo, monorreno congénito, con una insuficiencia renal avanzada; los estudios hematológicos confirman una gran identidad entre ambos y, como preparación previa, el paciente había sido sometido a una radiación completa hasta producir una total aplasia medular que se confirmó mediante una punción esternal, para lograr una "inmadurez inmunológica", con la abolición de la población linfocitaria, como se preconizaba en aquel momento. Tras la operación el riñón funcionó durante dos días pero el enfermo presentó un cuadro de hemorragia gene- ralizada que condujo al fallecimiento a la semana; el estudio necrópsico demostró una normalidad del riñón implantado, tal como expuso en la Reunión Nacional de la AEU en 1961 y al siguiente año en la publicación Medicamenta 8 (Fig. 4) y que recientemente ha rememorado el Dr. Virseda ${ }^{9}$ en la XXII Reunión del Grupo de Trabajo de Trasplante Renal, desarrollada en Murcia.

En el Hospital Clínico de la Universidad de Barcelona, dentro de la cátedra de Urología, se crea, en 1964, la «Unidad experimental de trasplante renal»; los nefrólogos, Drs. Caralps Riera y Alsina se entrenaron con una estancia en París ${ }^{10}$, junto al profesor Hamburger, en el Hospital PitiéSalpetrière, donde el urólogo Dr. René Küss efectuaba implantes renales desde 1952. Comienzan
Trasplante renal (homotrasplante entre padre e hijo) en un grave caso de insuficiencia renal en riñón único congénito

\section{POR LOS}

Doctores C. YOUNGER, A. HIDALGO, E. CHACON, R. RIVERA LOPEZ, J. PARRA, F. ARCE, A. CASAS, V. BARRIO, E. RAMIREZ TORRES, J. VISAS, J. CALDERON, B. ALVAREZ, B. MARTIN DE PRADO, F. SERRANO, I. FUENTES, E. PADILLA, M. BENARROT, R. ROS, J. RIVE. RA BANDRES, M. LOPEZ BRAVO, J. ELIO, J. FER \ANDEZ y A. PURAS Rvdo. P. JUAN MARIA DEL AMO Sres. J. M. CARDENAL y A. ESTANY Anesor eclesiastico Asesores jaridicos

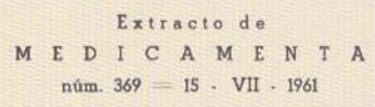

DrPostro LKGAL: M. Sep. $769 .-1958$.

M C M I X I

PUBLICACIONES DE LA DIRECCTON CIENTIFICA DEL INSTITUTO FARMACOLOGICO LATINO, S. A.
RLOS ROSAS, 87-TELEFONO 26363 O0 (6 LINEAS) MADRID
FIGURA 4. Trasplante Dr. Younger. 
sus trabajos en perros con la preparación de los líquidos para la conservación del riñón, a diferentes tiempos y temperaturas; la cirugía en el animal la realiza el Dr. D. José María Gil-Vernet, quien pone a punto su técnica de colocación ortotópica del órgano, proceder que publica en varias revistas internacionales ${ }^{11-15}$ (Figs. 5 y 6). Tras realizar un primer implante renal sin éxito en enero de 1965, lleva a cabo en el mismo año, el día 23 de julio, el segundo, en el que utilizó el de un varón de 24 años fallecido con traumatismo craneoencefálico en accidente de moto, a una mujer de 35 años diagnosticada de una nefroesclerosis en fase avanzada. El curso postoperatorio fue normal así como la función del injerto. Fue el primer trasplante renal con buena evolución y larga sobrevida del paciente y del órgano en nuestro país; en ese mismo año el equipo implantó otros ocho riñones más con éxito inmediato.

El siguiente grupo en lograr un resultado favorable fue el de la Clínica de la Concepción de Madrid; la intensa labor experimental llevada a cabo en el laboratorio de la Institución nos lo representa el hecho de que, cuando comienzan con los implantes renales en humanos, habian hecho 250 en animales, con todo los dos primeros homotrasplantes efectuados en el año 1964 por el Dr. Parra ${ }^{16}$, con riñón de cadáver, no son satisfactorios lo que hace que el Dr. D. Carlos Alférez Villalobos se marche a Estados Unidos para conocer la metodología y para ampliar su formación práctica; a su regreso con su equipo formado inicialmente por los Drs. García de la Peña y Vela Navarrete y el nefrólogo Dr. D. Luis Hernando Avedaño, lleva a cabo un implante renal en octubre de 1965, a una mujer de 21 años diagnosticada de pielonefritis crónica, a la que practican una nefrectomía bilateral y una esplenectomía como preparación previa y en la operación una anastomosis término terminal de la vena renal a la ilíaca y de la arteria renal a la hipogástrica con reimplante uretero vesical por medio de su técnica personal antirreflujo (Fig. 7); se obtiene una función renal con diuresis, pero a las 48 horas la

FIGURA 6. Técnica quirúrgica Dr. Gil Vernet. 


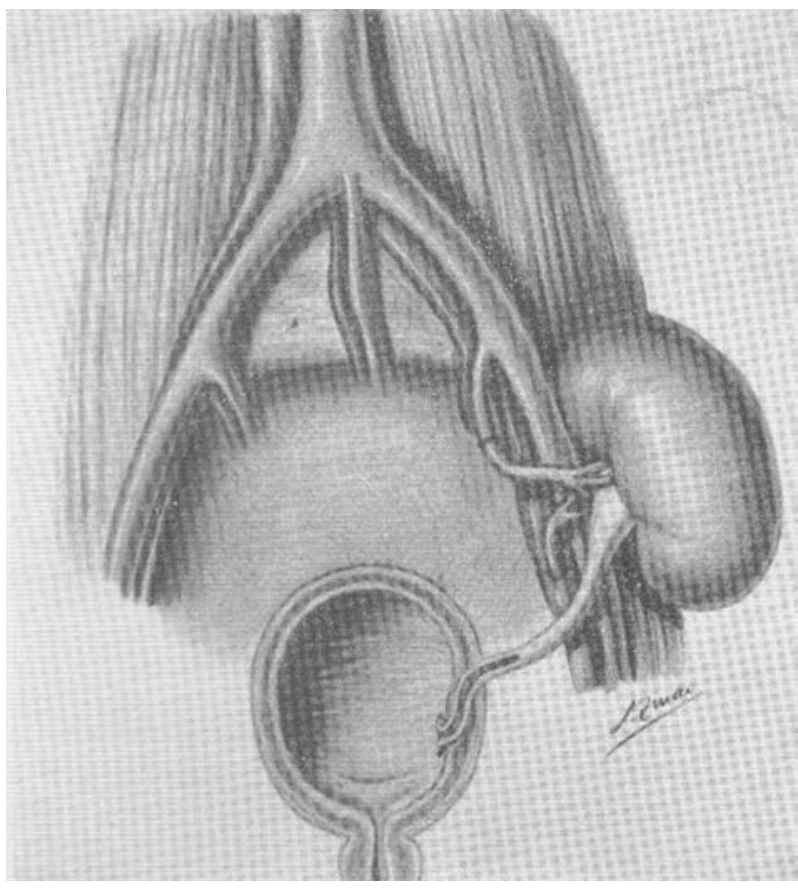

FIGURA 7. Técnica quirúrgica Dr. Alférez.

paciente fallece de una parada cardiorrespiratoria, comunican los pormenores en el VIII Congreso Luso Español de Urología celebrado en Oporto $^{17}$, en junio de 1969; efectúa el segundo implante el 24 de marzo de 1966 a un varón de 24 años aquejado de una nefropatía grave, con análogo procedimiento y buena evolución, sin complicaciones y prolongada sobrevida del injerto, caso que publica en 1967 en Archivos Españoles de Urología ${ }^{18}$ (Fig. 8).

Hemos de destacar que estos dos centros fueron pioneros en el estudio, en la investigación y en la experimentación para llevar a cabo los

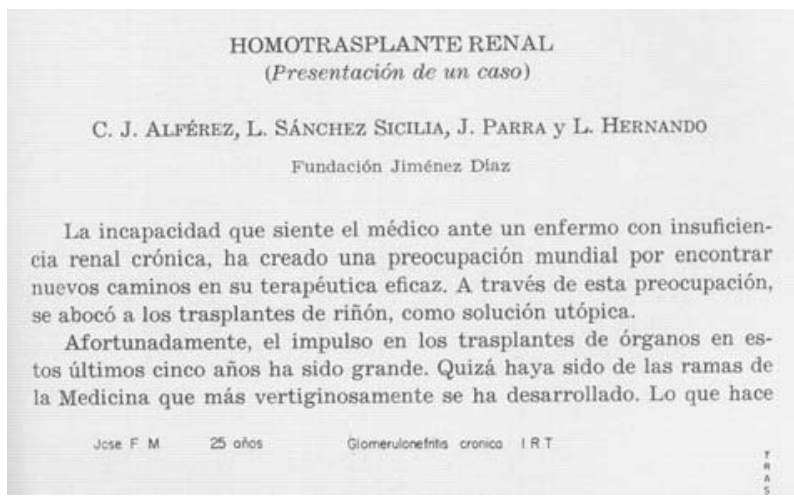

FIGURA 8. Comunicación trasplante Dr. Alférez. implantes, sin relación con la medicina oficial, al ser una técnica no reconocida, ni establecida oficialmente, ya que la legislación española del momento, que se regía por la ley de 18 de diciembre de 1950 y posterior normativa de 1955, que regulaba algunos limitados aspectos del trasplante de órganos, quedaba totalmente obsoleta y la administración sanitaria española no dio la menor importancia a los trasplantes. Surgieron grandes críticas de grupos y de estamentos que no aceptaban los trasplantes y en algunos ambientes médicos del país incluso desautorizaban el método, según el Dr. Gil-Vernet ${ }^{19}$, en la misma Real Academia de Medicina de Barcelona un ilustre académico dijo en una conferencia que el trasplante de riñón era una utopía y otro pidió a la Real Academia que desautorizara los trasplantes. Y esto consta en acta, lo que creó una gran polémica que pretendía despertar la conciencia de los políticos y de la sociedad. $\mathrm{El}$ problema fundamental lo presentaban la utilización de órganos de cadáver y el dictaminar a partir de qué momento se consideraba muerta una persona. Coincide con esta manifestación la realización del primer trasplante de corazón, en diciembre de 1967, por el Dr. Barnard (Fig. 9), que constituyó un fuerte revulsivo internacional al que siguió una intensa campaña de prensa a favor de los trasplantes en los países más adelantados de occidente; también el trasplante renal fue abordado como tema de estudio y defendido por el profesor Küss, en una mesa redonda, en el XIV Congreso de la Sociedad Internacional de Urología, celebrado en Munich en julio de 1967, y también en el X Congreso Americano de Urología, desarrollado en diciembre del mismo año ${ }^{20}$. Fue en 1968 cuando se estableció por el Comité de la Harvard Medical School el concepto de muerte cerebral aceptado internacionalmente, todo ello hizo que acallasen las críticas y se consolidaran definitivamente los trasplantes de órganos.

Tras estos primeros éxitos, introducidos los criterios de histocompatibilidad y la terapéutica con suero antilinfocitario, mejorada la técnica de extracción con la perfusión de los riñones con líquidos específicos de conservación y apoyados en los resultados que se obtienen y que refrenda la bibliografia internacional se prosigue con la labor trasplantadora, así el Dr. D. Pablo Carretero publica la 


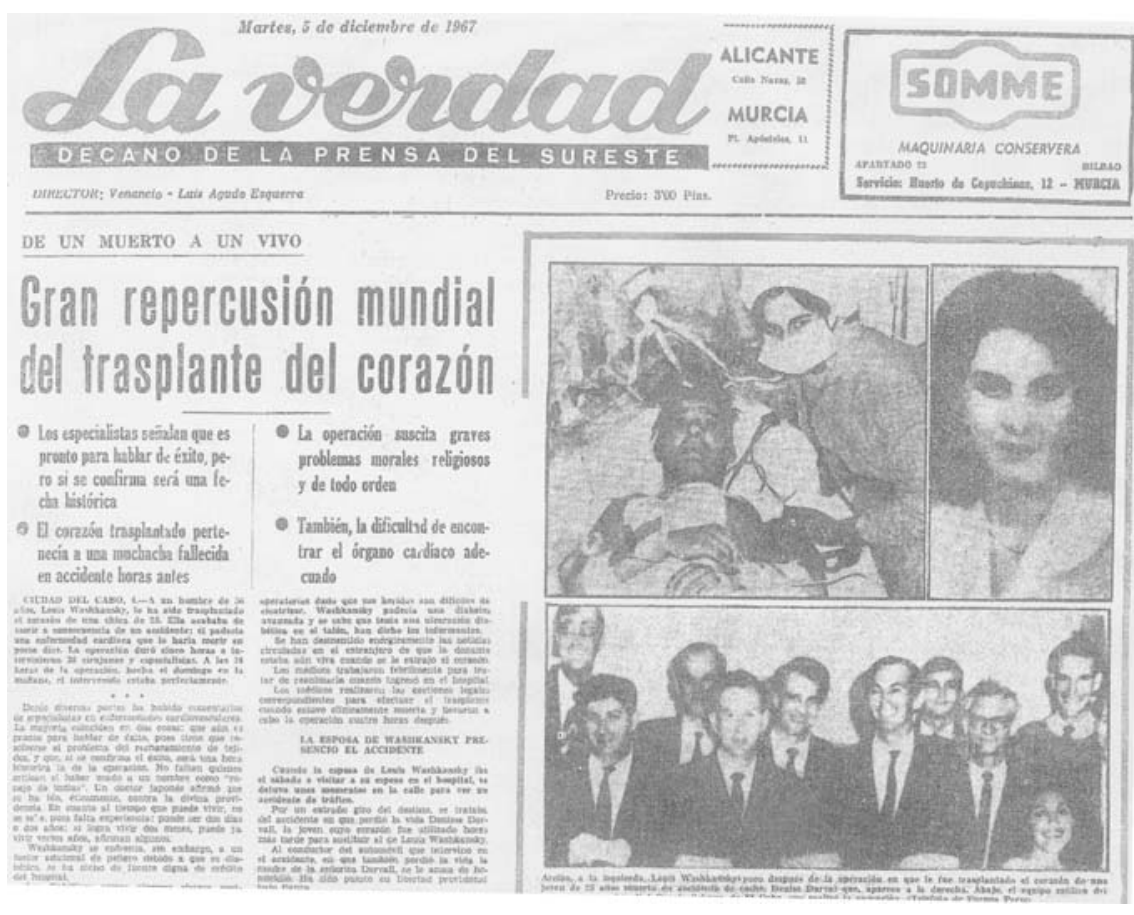

FIGURA 9. Repercusión en la prensa del primer trasplante de corazón.

La Clínica Puerta de Hierro de Madrid inicia su programa de trasplante renal en agosto de 1968, con una técnica análoga a la del centro anterior y con una anastomosis uretero-ureteral; hasta 1972 efectúan 15 implantes en 13 pacientes, 10 de cadáver y cinco de vivo, de los que diez años después viven ocho, con función normal de su riñón ${ }^{23,} 24$ (Fig. 12).

En esta década, que conozcamos, principia también su experiencia en 1966, en el Hospital de la Santa Cruz y de San Pablo de Barcelona, el Dr. Puigvert en el Instituto de Urología, con la colaboración del cirujano vascular Dr. Jurado Grau junto al Dr. Solé Balcells y al nefrólogo Dr. D. Gerardo del Río; efectúa un pri-

estadística del Hospital Clínico de Barcelona, entre 1965 y 1970, periodo en el que efectúan 45 implantes, de ellos siete son de vivo, 34 de cadáver y cuatro libres ${ }^{21}$ (Fig. 10) y en la que señala una mortalidad en los tres primeros meses del 33\%, en esta primera relación, que atribuye a la corta experiencia, a las elevadas dosis de inmunosupresión y a que hasta el año 1969 no se comenzaron a realizar las pruebas de histocompatibilidad, resultados que mejoran en la segunda serie, que comprende de 1970 a 1975, con 68 casos, en los que la mortalidad desciende al $8,7 \%$.

En la Clinica de La Concepción, de Madrid, desde octubre de 1965 hasta abril de 1969 habían llevado a cabo veinte trasplantes, con una evolución favorable y sobrevida superior al 50\% lo que les anima a proseguir $\mathrm{y}$, cuando ya tienen una suficiente experiencia, tras los diez primeros de cadáver, comienzan con el de donante vivo en enero de $1968^{22}$ (Fig. 11). mer implante sin resultado satisfactorio, lo que hace que nombren al Dr. D. Joaquín Ferré con formación trasplantadora en Estados Unidos como responsable de la Unidad quien prepara un laboratorio para realizar cirugía experimental en perros, primero, y, al año siguiente, 1968, en humanos; la evolución no fue la esperada por lo que el Dr. Puigvert cortó el programa, del que no era muy partidario, y no es hasta enero de 1980 cuando definitivamente el Dr. Villavicencio organiza la unidad de trasplante renal en la institución.

\begin{tabular}{|c|c|c|c|c|c|c|}
\hline \multicolumn{7}{|c|}{ GRUPO A (1965-1970) } \\
\hline & & \multicolumn{2}{|c|}{ Riñón de cadaver } & Riñón libre & & iñón de vivo \\
\hline & & \multicolumn{2}{|c|}{34} & 4 & & 7 \\
\hline $\begin{array}{l}\text { Eficacia pobre } \\
\text { Eficacia moderada }\end{array}$ & & $\begin{array}{r}16 \\
9\end{array}$ & & 3 & $81,8 \%$ & 6 \\
\hline $\begin{array}{l}\text { Eficacia buena } \\
\text { Eficacia muy buena }\end{array}$ & & $\begin{array}{l}2 \\
7\end{array}$ & $26,47 \%$ & 1 & $18,1 \%$ & 1 \\
\hline \multicolumn{4}{|c|}{ Mortalidad en los 3 primeros meses } & 0 & & \\
\hline & $\begin{array}{c}11 \\
(32,35 \%)\end{array}$ & \multicolumn{2}{|c|}{$\begin{array}{l}2 \text { hemorragias } \\
5 \text { sepsis } \\
1 \text { hepatitis } \\
3 \text { hemorr. digest. } \\
1 \text { parálisis p/intox. }\end{array}$} & & $\begin{array}{c}4 \\
(36,3 \%)\end{array}$ & $\left\{\begin{array}{l}2 \text { hemorragias } \\
1 \text { sepsis } \\
1 \text { hemorr. digest. }\end{array}\right.$ \\
\hline
\end{tabular}

FIGURA 10. Casuistica del H. Clínico de Barcelona, 1965-1970. 


\begin{tabular}{|c|c|c|c|c|c|c|}
\hline & & & TRAS: & WES DE R & & \\
\hline Nombre & Sexo & Hes & Año & Donsinte & $\begin{array}{l}\text { Teontos } \\
\text { isacular }\end{array}$ & $\begin{array}{l}\text { Bontca } \\
\text { ureteral }\end{array}$ \\
\hline$X \cdot D \cdot A \cdot A$. & H & Ootubre & 1965 & Cadáver & Il1achipog. & uréter-vejiga \\
\hline J.R.Y. & $\vec{v}$ & Marzo & 1966 & Codáver & Illao.hipog. & uréter-vej1ga \\
\hline S.M.S. & H & Abril & 1966 & Cadáver & Illac.H1pog. & uréter-vejiga \\
\hline$\ldots$ & - & Ootubre & 1966 & Cadáver & Illac.hipog. & uréter-vejiga \\
\hline M.K.A. & H & Kayo & . 1966 & Cadáver & Illac.h1pog. & uréter-vejiga \\
\hline K・I.D.S. & म & Noviem. & . 1966 & Cadáver & Il1ac.h1pog. & uréter-vejiga \\
\hline A.S?.S. & v & Marzo & 1967 & Cadáver & Ilise.h1pog. & uréter-vejigs \\
\hline C.L.S. & H & Yarzo & 1967 & Cadáver & Iliac hipog. & uréter-vejiga \\
\hline J.G.P. & v & Mayo & 1967 & Cadáver & Iliachipog. & uréter-vejiga \\
\hline Y०E.G.M. & H & Noviem. & . 1967 & Cadáver & Il tao.hipog. & uréter-vejiga \\
\hline E.C. & B & Enero & 1968 & Hermans & Cavs-hipog. & uréter-vejiga \\
\hline-- & - & Junio & 1968 & Cadáver & Illsc.h1pog. & uréter-vojiga \\
\hline-- & - & Pebrero & 1969 & Cadáver & Cava-hipog. & uréter-vejlga \\
\hline S.B.I. & H & Mayo & 1968 & Hermana & Cava-hipog. & pelvis-pelvis \\
\hline J.S. & $\nabla$ & Julio & 1968 & Hermana & Cava-hipog. & pelvis-pelvis \\
\hline X.R.P. & H & Diciom. & 1968 & Cadáver & Cava-hipog. & pel vis-pelvis \\
\hline A.B.M. & $\mathrm{v}$ & Pebrero & 1969 & Cadáver & Cavs-h1pog. & pelvis-pelvis \\
\hline J.M.G. & v & Febrero & 1969 & Cadáver & Cava-hipog. & pelvis-pelvis \\
\hline R.G.M. & $\nabla$ & Marzo & 1969 & Ввровя & Cavs-hipog. & pel vis-pelvis \\
\hline A.R.T. & v & Abril & 1969 & Padre & Cava-hipog. & pelvis-pelvis \\
\hline
\end{tabular}

FIGURA 11. Casuistica Clínica de la Concepción, 1965-1969.

Entre agosto de 1968 y septiembre de 1972, se realizaron en la Clínica Puerta de Hierro 15 trasplantes renales (T.R.) en 13 pacientes. De

TABLA I

\begin{tabular}{|c|c|c|c|c|c|c|c|}
\hline Caso & Fecha & Sexo & Edad & Donante & $\begin{array}{l}\text { Id. } \\
\text { HLA }\end{array}$ & T. isqu & uemia \\
\hline 1 & $06-69$ & M & 20 & Hermana & 4 & $38 \mathrm{n}$ & $\min$. \\
\hline 2 & $11-69$ & V & 20 & , & 4 & 45 & $n$ \\
\hline 3 & $12-69$ & V & 22 & Cadáver & 2 & 50 & = \\
\hline 4 & $05-70$ & $M$ & 25 & Madre & 2 & 107 & \\
\hline 5 & $11-70$ & V & 27 & n & 2 & 47 & " \\
\hline 6 & $05-71$ & V & 36 & Cadáver & 2 & 175 & " \\
\hline 7 & $03-72$ & V & 21 & " & 2 & 141 & " \\
\hline 8 & $09-72$ & M & 26 & " & 2 & 167 & $=$ \\
\hline
\end{tabular}

FIGURA 12. Casuistica Clinica Puerta de Hierro.

De la bibliografía de esta década, destacamos el discurso de ingreso en la Real Academia de Medicina de Murcia del urólogo D. Juan Martínez García, en 1966, sobre Trasplante renal ${ }^{25}$, en el que recoge detalles de los seis implantes llevados a cabo en España hasta el momento y, en 1967, la tesis doctoral del Dr. Serallach Nuevo tratamiento de la hipertensión vásculorenal "el autotrasplante renal" 26 , procedimiento que preconiza y realiza por vez primera en nuestro país. Se comienzan, en 1968, a presentar ponencias sobre trasplante renal en los cursos de postgrado, así en el V Curso Internacional de Urología organizado por los Drs. Gil-Vernet y Gregoire, en el Hospital Clínico de Barcelona (Fig. 13), en el mes de octubre, el Dr. Küss habla del «Estado actual de la homotrasplantación renal" y el profesor Gil-Vernet de "Aspectos quirúrgicos del trasplante renal $»^{27}$.

\section{DÉCADA DE LOS SETENTA}

La Asociación Española de Urología, dentro de su programa de actividades científicas, celebra en abril de 1970 una sesión clínica sobre "Trasplantación renal" (Fig. 14), en la que, además de los profesores extranjeros, participan los Drs. GilVernet, Alférez Villalobos, Casimiro Romero y Solé Balcells $^{28}$. En julio de 1976 tiene lugar el XLI Congreso Nacional de Urología en León, donde el Dr. Alférez Villalobos presenta su ponencia sobre

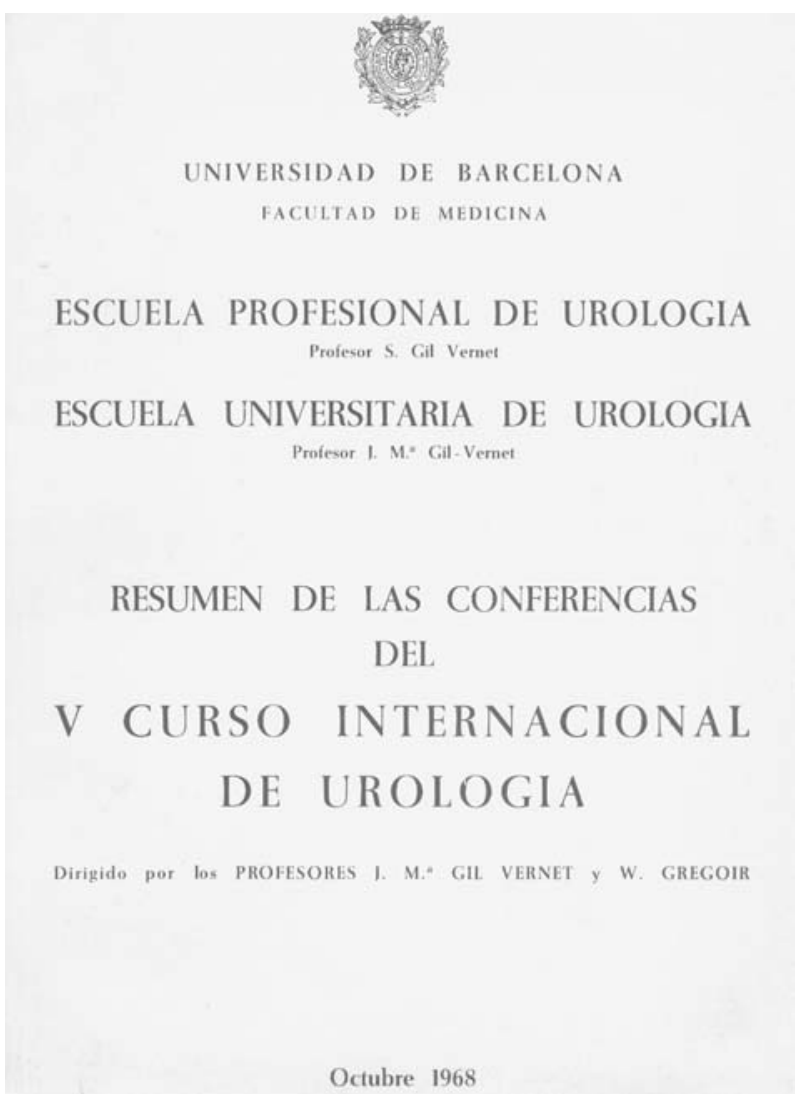

FIGURA 13. V Curso Internacional de Urologia. 
ASOCIACION ESPANOLA DE UROLOGIA

CURSO $1969-1970$

ACTIVIDADES CIENTIFICAS

Dia 18 de abril de 1970 (sábado, once mafiana): Trasplantación renal.

Histocompatiblidad. Prof. Traeger, catedrático de ta Universidad de Lyon.

Elección del donante, preparación y conservación del injerto. Prof. Toussaint,

jefe del Departamento de Nefrologia del Hospital Brugmann, de Bruxelles,

Técnica quirúrgica. Comprobaciones. Prof. José Maria Gu Vernet, profesor de Urologia de la Facultad de Medicina de Barcelona.

\section{FIGURA 14. Sesión científica de la AEU.}

Trasplantes de riñón ${ }^{29}$, que resume perfectamente la situación que tienen en ese momento en nuestro país; todo ello da lugar a que en el año 1978 se organice en el seno de la AEU el «Grupo de Trabajo de Trasplante Renal", bajo la coordinación del Dr. Alférez, quien permanecerá en el cargo por espacio de diez años. La primera reunión, casi familiar, se desarrolló en el Hotel Mindanao de Madrid en 1979, con la presencia, que sepamos, de los Drs. Alférez, Páramo, Lobaco, Allona y Berenguer y sin que conozcamos pormenores de su desarrollo. Cuando ya la pujanza y la dinámica de los grupos se establecen, se acuerda una rotación en el puesto cada cuatro años, le sucede el Dr. Serrallach y los sucesivos coordinadores del Grupo (Tabla 1).

\section{Tabla 1}

Grupo de Trasplante Renal de la AEU. Coordinadores

\begin{tabular}{ll}
\hline Dr. Alférez Villalobos, C. & $1978-1988$ \\
Dr. Serrallach Milá, N. & $1988-1992$ \\
Dr. González Martín, M. & $1992-1996$ \\
Dr. Burgos Revilla, F. J. & $1996-2000$ \\
Dr. Montañés Medina, P. & $2000-2004$ \\
Dr. Alcaraz Asensio, A. & 2004
\end{tabular}

En mayo de 1970, en el Hospital Clínico de Barcelona, el Dr. Gil-Vernet, en el I curso sobre Tratamiento de la Uremia terminal ${ }^{30}$, trata del "Trasplante en sus aspectos inmunológicos y quirúrgicos" y en junio del mismo año y en la misma ciudad tiene lugar el II curso de Técnica quirúrgica de los trasplantes, organizado por la unidad de cirugía experimental del Hospital San Pablo de Barcelona, adscrito al programa de postgrado de la Universidad Autónoma, con la participación del equipo de trasplantes del Hospital Clínico catalán, con el tema "Aspectos médicos y quirúrgicos del trasplante renal en el hombre»31; en el Congreso de la Sociedad Internacional de Urología celebrado en Tokio, en 1970, en el que fue elegido presidente de la misma el Dr. D. Salvador Gil Vernet, se trata en una mesa redonda de la "Preservación y conservación renal»"32 y en abril de 1978, en la cátedra de Urología de la Universidad de Barcelona, se imparte el I Curso Internacional de Trasplante renal dirigido por el Dr. Gil-Vernet ${ }^{33}$.

La actividad trasplantadora en los hospitales continúa, en 1970 el Dr. Martínez Piñero la reanuda en el Hospital La Paz, de Madrid, junto con el Dr. Sánchez Sicilia ${ }^{34}$, quienes, desde esta fecha hasta 1976, efectúan once implantes, seis de cadáver y cinco de vivo, con una mortalidad quirúrgica nula, recogemos de la ponencia del Dr. Alférez (Fig.15) estas cifras estadísticas así como las del Hospital Militar Gómez Hulla, en donde el Dr. Acero Santamaría, al frente de la unidad renal del centro, con los Drs. Diz y Quiroga inicia los trasplantes en 1971 y en los cuatro años siguientes realizan siete de donante cadáver.

Hacia mitad de la década la red hospitalaria de la Seguridad Social se expande y cubre las principales poblaciones del país, comienzan a

Dres.: J. A. Martinez Piñeiro y L. Sánchez Sicilia. Han realizado 11 trasplantes, de los cuales 6 han sido de donante cadáver y 5 de vivo, correspondiendo 2 a donante padre, 2 a donante madre y 1 de hermano.

$\mathrm{La}$ mortalidad quirúrgica ha sido $\mathrm{O}$, viviendo con rinón funcionante 2 pacientes.

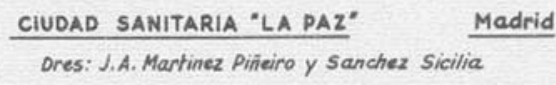

Supervivencia cumulativa del injerto
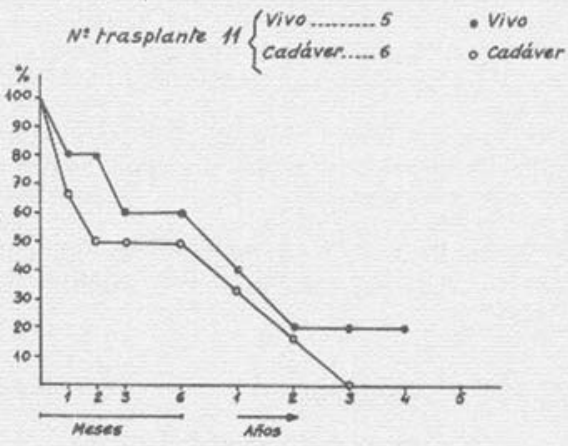

FIGURA 15. Casuistica H. La Paz, 1970. 
establecerse servicios de Nefrología con la práctica de la hemodiálisis, la cual se prescribe ya a todos los pacientes con insuficiencia renal crónica; el paulatino incremento de estos en edades tempranas, junto a la proliferación de estas unidades, fue una de las causas que contribuyó en mayor medida a que se desarrollaran los equipos quirúrgicos de trasplante renal y así, poco a poco, se organizan e incorporan a este programa los servicios de Urología de los principales nosocomios españoles, como el Hospital Universitario Marqués de Valdecilla, de Santander, en febrero de 1975, con el Dr. Abaitúa Bilbao, apoyado por el servicio de cirugía vascular, que por un largo periodo colaborará en la sutura de los vasos ${ }^{35}$; en un año efectúan ocho implantes, seis de donante cadáver y dos de vivo.

En Madrid, en el Hospital Clínico, el profesor D. Hipólito Duran junto con los miembros del servicio de Urología llevan a cabo en 1975 su primer implante renal; el Hospital 12 de Octubre comienza su actividad trasplantadora en septiembre de 1976; los miembros del servicio de Urología con el Dr. Leiva como responsable junto con los Drs. Polo, Díaz González y Fraile ${ }^{36}$, quienes colocan un riñón de cadáver, extraído en el propio centro, a un varón de 28 años con una evolución satisfactoria, no iniciaron los de donante vivo hasta julio de 1984. El Hospital Gregorio Marañón, en septiembre de 1975, se incorpora al programa y en 1988 nos presenta las complicaciones que han tenido cuando han efectuado 28037; en noviembre de 1979 lo hace el Hospital Ramón y Cajal que es el último de este periodo en agregarse al programa y relatan, en la publicación en 1986, los problemas vasculares que han tenido ${ }^{38}$.

En Barcelona, el servicio de Urología del Hospital del Valle de Hebrón, dirigido por el Dr. Solé Balcells, nombra responsable del equipo quirúrgico al Dr. Quintanilla, quien, tras un periodo de cirugía experimental en perros, comienza en 1976 los implantes renales en humanos, también lo hace en 1979 el Hospital de Ntra. Sra. de la Esperanza, de Barcelona, con el Dr. Ballesteros Sampol como director de la unidad en la que efectúan el reimplante pielo-pielico ${ }^{39}$.

La Clínica Universitaria de Navarra principia en noviembre de 1976 con el Dr. Berián Polo, quien dirige el equipo, y en 1985 publica un estudio retrospectivo de 101 implantes en el que recoge su serie actuarial ${ }^{40}$. En el Hospital Infantil La $\mathrm{Fe}$, de Valencia, el Dr. García Ibarra es el primero en establecer un programa de trasplante renal en niños en $1978^{41}$. En el Hospital de Cruces, en Baracaldo, los Drs. Bernuy y Pertusa arrancan en julio de 1979 con la práctica del trasplante renal, datos que entresacamos de su artículo sobre la relación entre el trasplante y la situación laboral del paciente ${ }^{42}$.

En Andalucía los servicios de Urología de cuatro centros son los que se incorporan a realizar trasplantes renales, en abril de 1978 lo hace el Hospital Virgen del Rocío, de Sevilla, con el Dr. Montañés al frente, cuya casuística expone en la ponencia sobre trasplante renal que efectúa en $1992^{43}$ (Fig. 16). En Córdoba, el grupo del Hospital Reina Sofia ${ }^{44}$, dirigido por el Dr. López López, introduce el proyecto en enero de 1979, a la vez que el Hospital Carlos Haya, de Málaga ${ }^{45}$, con el Dr. Gutiérrez Calzada a la cabeza. El grupo del Dr. Zuluaga, del Hospital Clínico de Granada, realiza el primero, el 27 de abril de 1979, de donante vivo entre hermanos gemelos ${ }^{46}$ (Tabla 2).

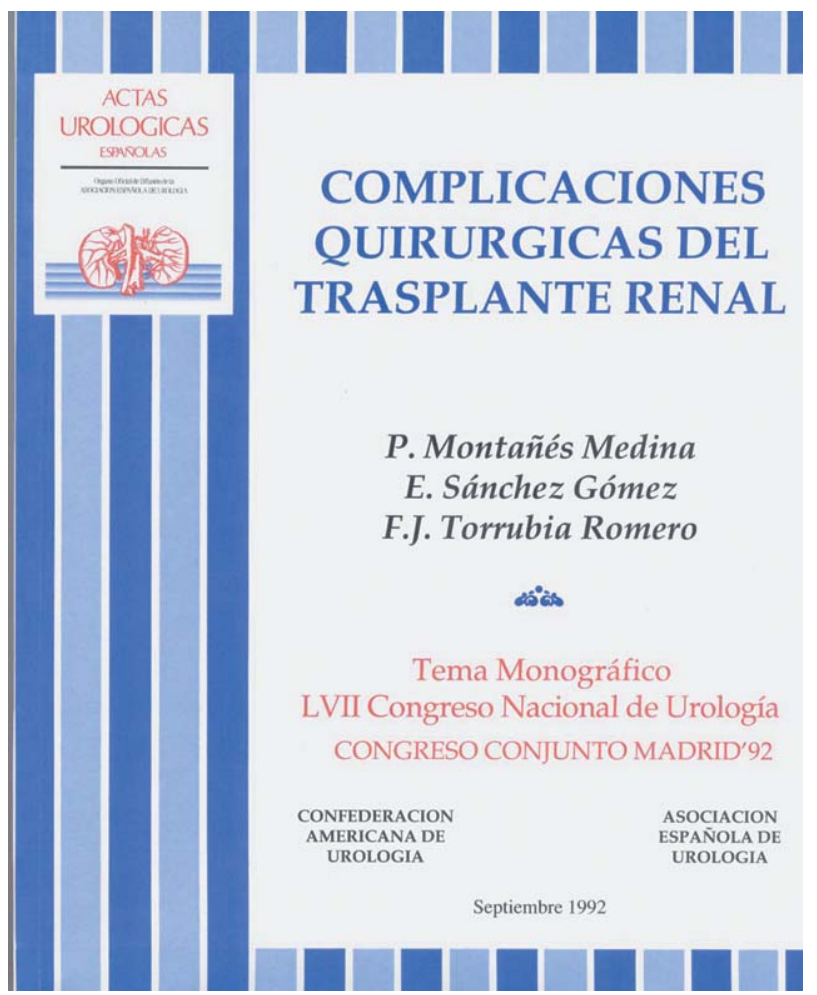

FIGURA 16. Ponencia de Trasplante renal Dr. Montañés. 
Tabla 2

Incorporación de Hospitales. Década de 1970

\begin{tabular}{ll}
\hline 1976 (sep.) & $\begin{array}{l}\text { H. } 12 \text { de Octubre, Madrid } \\
1976 \text { (sep.) }\end{array}$ \\
1976 (nov.) & $\begin{array}{l}\text { H. Ramón y Cajal, Madrid } \\
\text { Clínica Universitaria de Navarra, } \\
1976 \text { (nov.) }\end{array}$ \\
$\begin{array}{l}\text { Pamplona } \\
1976\end{array}$ & H. del Valle Hebrón, Barcelona \\
1978 (abril) & H. Virgen del Rocío, Sevilla \\
1978 & H. Infantil La Fe, Valencia \\
1979 (enero) & H. Reina Sofia, Córdoba \\
1979 (enero) & H. Carlos Haya, Málaga \\
1979 (abril) & H. Clínico de Granada \\
1979 & H. de Cruces, Baracaldo. Vizcaya \\
\hline
\end{tabular}

Entre la bibliografia de este periodo resaltamos, en 1973, dos trabajos de los Drs. Diz y Anitúa, del Hospital Militar Gómez Ulla, sobre su experiencia en la técnica de extracción renal ${ }^{47-48}$, destacamos las aportaciones del grupo del Hospital Clínico de Barcelona en el Congreso Nacional de Urología de 1976, donde presentan siete comunicaciones en las que resumen su actividad cuando llevan realizados 67 implantes $^{49-55}$, y en la reunión de la octava regional de la AEU en 1977 el Dr. Berián, de la Clínica Universitaria de Navarra, presenta un programa para la organización de los trasplantes de riñón ${ }^{56}$.

La determinación de la histocompatibilidad de los tejidos se lleva a cabo ya de modo sistemático son dos, en Madrid, los laboratorios nefrológicos que las efectúan, lo que da lugar a que los hospitales se agrupen en torno a uno de ellos y, así, a mediados de la década se establecen el "Centro trasplante" y el "Madrid trasplante", con una lista común entre los hospitales adscritos a cada uno para el intercambio de órganos, de modo que se pudiera implantar a la persona más idónea de toda la relación disponible en el laboratorio, la última organización posteriormente se extenderá a algunas poblaciones de las Comunidades Autónomas cercanas. Esta situación de la aparición de entidades reguladora de los intercambios se crea también a nivel internacional, en 1979, el Eurotrasplante.

\section{DÉCADA DE LOS OCHENTA}

La promulgación de la ley de española de trasplante data del 27 de octubre de 1979, y su pos- terior desarrollo en el Real Decreto de 1980, vino a llenar el vacío legal existente en materia de extracción y de trasplante de órganos; fue una de las más adelantadas internacionalmente en su momento debido a su criterio de reconocimiento de la muerte cerebral, fue la clave para el despegue definitivo de los trasplantes renales y para la puesta a punto paulatina de los equipos y dictó las normas sobre la infraestructura y los medios que debían reunir los hospitales para acreditarlos y poder llevarlos a cabo. En Cataluña, en 1982, se crea el "Registro de pacientes con insuficiencia renal", la lista de espera para recibir un trasplante se incrementa paulatinamente debido a la falta de órganos; en el Congreso Francés de Diálisis y Trasplante, en dicho año, se pone de manifiesto el papel que desempeñan los coordinadores y su eficacia para la obtención de donantes; el departamento de Sanidad de la Generalitat instituye la figura del coordinador autonómico en 1984, a raíz de iniciarse los trasplantes de hígado y cardíacos ya que la localización de un receptor adecuado para estos órganos era dificultosa y de ese modo se facilitaba el contacto entre el centro coordinador y el extractor además de poner a su disposición los medios de transporte necesarios, avión, ambulancias, etc., para el desplazamiento de los grupos para la extracción; en poco tiempo se demuestra la efectividad del procedimiento y la conveniencia de disponer en cada hospital de una persona dedicada a esta misión, con lo que nacen los coordinadores hospitalarios y se crea la Oficina de Intercambio de Órganos de la Generalitat de Cataluña, en 1985, que fue el germen para el desarrollo posterior de la Organización Nacional de Trasplante (ONT).

Tras la promulgación de la ley de Seguridad Vial, con la obligatoriedad de llevar el casco puesto los motoristas, se reducen los accidentes con traumatismo cráneo-encefálico, principal fuente de obtención de órganos hasta ese momento, a lo que se suma la huelga general de médicos del año 1987, con lo que disminuyó el número de trasplantes en todo el país de modo significativo en los dos años siguientes; tuvo una gran repercusión en la opinión pública y determinó una actuación del Defensor del Pueblo, tras hacerle llegar las asociaciones de enfermos y de familiares, su protesta por este descenso. La Organización Nacional 
de Trasplante, dependiente del Ministerio de Sanidad, estaba creada por Real Decreto de 22 de febrero de 1980, con la finalidad de coordinar todas las actividades relacionadas con los trasplantes en general, potenciar su práctica, gestionar los intercambios y cooperar con las distintas Comunidades Autónomas, pero no fue dotada de estructura y personal hasta la intercesión mencionada. A finales de 1989 la ONT se estructura adecuadamente, acorde con nuestras características sanitarias, y se proyectan los equipos de coordinación autonómica y hospitalaria. El primer coordinador nacional fue el Dr. D. Ángel Zamarrón al que sustituyó un año más tarde el Dr. D. Rafael Matesanz ${ }^{57}$.

En enero de 1980 la Fundación Puigvert inicia su programa de trasplante renal, que dirige el Dr. Villavicencio ${ }^{58}$. En Valencia, en el Hospital La Fe, el servicio de Urología con el Dr. Jiménez Cruz, lo hace en el mes de febrero ${ }^{59}$. El Hospital Príncipes de España, y actual de Bellvitge, en Hospitalet de Llobregat, con el Dr. Serrallach al frente, inicia su experiencia en marzo de ese $\mathrm{año}^{60}$. El Dr. González Martín, en el Hospital Juan Canalejo de La Coruña, principia su serie en enero de $1981^{61}$, al igual que el Hospital Universitario de Tenerife, en la Laguna, con el Dr. Bañares Baudet ${ }^{62}$; les siguen en 1982, los servicios de Urología del Hospital General de Galicia, en Santiago de Compostela, con el Dr. Cimadevila Covelo ${ }^{63}$, y el Hospital Universitario Puerta del Mar, de Cádiz, con el Dr. Romero Tenorio ${ }^{64}$. Dos años más tarde, en julio de 1984, el Hospital Universitario Virgen de la Arrixaca, de Murcia, comienza los implantes renales, con la supervisión del Dr. Server, tras tres años de tener concedida autorización como centro extractor de órganos ${ }^{65}$. El Hospital General de Asturias lo hace en el mismo año con el Dr. Muruamendiaraz como responsable del equipo $^{66}$. El Hospital Trías y Pujol, de Badalona, con el Dr. Saladie Roig se estrena en 1985; el Hospital Miguel Server, de Zaragoza, en junio de 198667; el Hospital Son Dureta, de Palma de Mallorca dirigido por el Dr. Ozonas, sin poder precisar la fecha, son en 1988, el Hospital General de Alicante, con el Dr. Mira Llinares y el Hospital Virgen de las Nieves de Granada, bajo la dirección del Dr. Tallada ${ }^{69}$, que sustituye en dicha población al del Hospital Clínico del Dr.
Zuluaga que finaliza su programa y los últimos en incorporarse en esta década son el Hospital Infanta Cristina, de Badajoz y el Complejo Hospitalario de Salamanca (Tabla 3).

Tabla 3

Incorporación de Hospitales. Década de 1980

\begin{tabular}{ll}
\hline 1980 (enero) & Fundación Puigvert, Barcelona \\
1980 (enero) & H. La Fe (adultos), Valencia \\
1980 (marzo) & H. Bellvitge, Barcelona \\
1981 & H. Juan Canalejo, La Coruña \\
1882 & $\begin{array}{l}\text { H. General de Galicia, Santiago de } \\
\text { Compostela }\end{array}$ \\
1982 & H. Puerta del Mar, Cádiz \\
1984 (julio) & H. Virgen de la Arrixaca, Murcia \\
1985 & H. Gérman Trías y Pujol, Badalona \\
1986 (junio) & H. Miguel Servet, Zaragoza \\
1987 & H. Infantil La Paz \\
1988 (junio) & H. General de Alicante \\
1988 (oct.) & H. General de Asturias, Oviedo \\
& H. Son Dureta, Palma de Mallorca \\
& H. Infanta Cristina, Badajoz \\
& Complejo Hospitalario de Salamanca \\
\end{tabular}

El Grupo de Trabajo de Trasplante Renal de la Asociación Española de Urología queda configurado en estos años, se establece una reunión anual a la que se agregan los componentes de las nuevas unidades de trasplante y en la que se presentan comunicaciones con la experiencia tenida en sus series, complicaciones, aparición de tumores, situaciones complejas, evolución, resultados, se aportan trabajos experimentales, etc., con lo que se afianza como medio de intercomunicación entre los diferentes centros trasplantadores (Tabla 4).

\section{DÉCADA DE LOS NOVENTA}

La labor realizada a partir de estos años es conocida por todos, prosigue la inclusión de centros hospitalarios con la determinante de que en todos ellos son los servicios de Urología los únicos responsables del acto quirúrgico, se completa la cobertura de todo el territorio nacional y se produce un aumento sostenido y progresivo tanto 
Tabla 4

Reuniones del grupo de trabajo de la AEU. Años 80 .

\begin{tabular}{|c|c|c|}
\hline II & 1980 & Valencia \\
\hline III & 1981 & Fuengirola \\
\hline IV & 1982 & Baqueira Beret \\
\hline $\mathbf{v}$ & 1984 & Cádiz, 24-25 febrero. Organizador Dr. Flores Ginés. \\
\hline VI & 1985 & Sevilla, Organizador Dr. Montañés Medina. \\
\hline VII & 1986 & $\begin{array}{l}\text { Bilbao, 16-18 de marzo. Organizador Dr. Bernuy Malfaz. Temas: "Valoración estadística y resultados } \\
\text { de los centros de trasplante», "Trasplantectomía y complicaciones urológicas del trasplante». }\end{array}$ \\
\hline VIII & 1987 & $\begin{array}{l}\text { Andorra, } 27-30 \text { de enero. Organizador Drs. Serrallach Mila y Cardelus. } \\
\text { Temas: "Métodos de exploración del enfermo trasplantado", "Factores de riesgo en el trasplantado } \\
\text { renal", "Cirugía previa y consecutiva al trasplante renal. "Estadística general del trasplante renal. }\end{array}$ \\
\hline $\mathbf{I X}$ & 1988 & $\begin{array}{l}\text { Madrid, 25-26 de noviembre. Reunión Científica de la AEU. Prof. Dubernard "Complicaciones del } \\
\text { trasplante renal" y "Trasplante renal y pancreático". }\end{array}$ \\
\hline $\mathbf{x}$ & 1989 & $\begin{array}{l}\text { Sierra Nevada, Granada, } 23-26 \text { de enero. Organizador Dr. Tallada Buñuel. } \\
\text { Temas: "Obtención de órganos: aspectos técnicos y médico legales", "Trasplante renal en portadores de } \\
\text { patología del aparato urinario inferior". }\end{array}$ \\
\hline
\end{tabular}

de las extracciones, ya prácticamente todas multiorgánicas, como de los implantes, se consolidan los trasplantes simultáneos de riñón con el páncreas, de los que el Hospital Clínico de Barcelona fue el pionero en $1983^{70}$, se inician los hepatorenales en 1993 y los reno-cardíacos; se incrementa paulatinamente la edad del donante a la par que la del receptor, se buscan donantes en asistolia y se efectúan implantes dobles de riñones añosos.

Todo este movimiento dio lugar a que se estableciera la llamada "Cultura del trasplante» entre los profesionales sanitarios con un paulatino entusiasmo por aumentar el número de implantes y conseguir mejorar los resultados, a lo que contribuyó muy eficazmente el establecimiento de los coordinadores hospitalarios como arma para la obtención de los órganos donados y España se afianza desde 1992 como el primer país del mundo en la obtención de órganos y en el número total de trasplantes de riñón efectuados anualmente, la expresión "Modelo Español" reconoce internacionalmente esta labor, cuyo éxito es debido a la vocación, entrega, sacrificio y preparación de los componentes de los equipos de trasplante.

\section{REFERENCIAS}

1. Rotellar E. 25 Años de hemodiálisis. Sociedad Española de diálisis y trasplante (SEDYT), 1982, IV(2):47-52.
2. Tramoyeres A, Lorca García J. Fallo renal agudo por intoxicación barbitúrico aguda, recuperado por aplicación de riñón artificial. Reunión Anual de la AEU, Valencia, mayo 1961.

3. Vela Navarrete R. Trasplantes renales. Consideraciones históricas. Ponencia Congreso Nacional AEU, Actas Asoc Esp Urol. 1976;VIII(1):9-11.

4. Parra J, Torres JA, Álvarez Rivas J, Albert C, Oliva H, et al. Cumulative production of drug-irradiation chimera as a method of inducing tolerance for homotransplantation of kidney. Ann N Y Acad Sci. 1962;99:781-786.

5. Calne RY. Renal transplantations, $2^{\mathrm{a}}$ ed. Arnold, London, 1967.

6. Vela Navarrete R. Mecanismo de rechazo del homoinjerto renal. Rev Clin Esp. 1965;99(1):14-28.

7. Martínez-Piñero JA. Trasplantación renal entre gemelos homocigóticos. Rev Clin Esp. 1961;82(2):83-92.

8. Younger C, Hidalgo A, Chacón E, Rivera López R, Parra J, Arce F, et al. Trasplante renal (homotrasplante entre padre e hijo) en un grave caso de insuficiencia renal en riñón único congénito. Medicamenta. 1961;VII(369):15-32.

9. Virseda Rodríguez J. 1961: El segundo trasplante renal en España (un largo camino histórico). Póster XXII Reunión Grupo Trasplante Renal de la AEU, Murcia, marzo de 2005.

10. Caralps A. Gil Vernet JM, Vives J, Andreu J, Brulles A, Tornos D, et al. Trasplante renal, 1983, Edit. Toray S. A., Barcelona

11. Gil-Vernet JM. Homotrasplantation renale. Journal d'Urologie. 1967;75:86-92.

12. Gil-Vernet JM.Human renal homotransplantation with cadaver kidneys. Technical summary. Results. Acta Urol Belg. 1967;35(1):86-91.

13. Gil-Vernet JM, Caralps A. Human renal homotransplantation. New surgical tecnique. Urol Int. 1968;23(3):201-204.

14. Gil-Vernet JM. Homotrasplantation renale. Journal Urol Nefro. 1969;75(12):221-227. 
15. Gil-Vernet JM, Gil-Vernet A, Caralps A, Carretero P, Talbot-Wriht $\mathrm{R}$, et al. Orthotopic renal transplant and results in 139 consecutive cases. J of Urol. 1989;142(1): 248-250.

16. Martínez García J. Trasplante renal, Memoria de ingreso en la Real Academia de Medicina de Murcia, pág. 57, 1966, Murcia

17. Alférez C. Trasplantes de riñón. Congreso Luso Español de Urol, Oporto, 1969. Actas Asoc Esp Urol. 1969;I(3):63-66.

18. Alférez C, Sánchez Sicilia L, Parra J, Hernando L. Homotrasplante renal (Presentación de un caso). Arch Esp Urol. 1967;XX(1):23-30.

19. Ruiz de Villalobos CL. Artífices hace cuarenta años del primer trasplante renal funcionante en España. Noticias Médicas 2005;3877:43-47.

20. Pérez Castro E. XIV Congreso de la Sociedad Internacional de Urología, Munchen, 9-14 de julio de 1967. Arch Esp Urol. 1967,XX(3):356-357.

21. Carretero P, Ballesteros A, López J, Brulles A, Figuls S, Rosas S, et al. Experiencia en 113 casos de trasplante renal. Actas Urol Esp. 1977;1(4):185-188.

22. Alférez C. Trasplantes de riñón", Congreso Luso Español de Urol, Oporto, 1969, Actas Asoc Esp Urol. 1969;I(3).

23. Egido de los Ríos J, Casado Pérez S, Alférez, C. Supervivencia de los trasplantes renales en la Fundación Jiménez Díaz, Congreso Español de Urol, León, 1976, Actas Asoc Esp Urol 1976;VIII(1):84-86.

24. Fernández Fernández J, Sanz Guajardo D, Gallego JL. Trasplantes renales de larga evolución. SEDYT. 1981;III (1):53-56.

25. Martínez García J. Trasplante renal. Memoria de ingreso en la Real Academia de Medicina de Murcia, 1966, Murcia.

26. Serrallach Mila N, Paravisini J, Albert J, Mayol Valls P, Casellas P, Torner Soler A, et al. Renal autotransplantation. Lancet. 1965;27:1130-1131.

27. Gil-Vernet JM, Gregoire W. V Curso Internacional de Urología", 1968, Barcelona.

28. Asociación Española de Urología. Actividades Científicas, Arch Esp Urol. 1970;XXIII (1): 92.

29. Alférez C. Trasplantes de riñón. Ponencia, XLI Congreso Nacional de Urol, León, 1976, Actas Asoc Esp. Urol. VIII(1).

30. Gil-Vernet JM. I curso sobre "Tratamiento de la uremia terminal”, 1970, Barcelona, Arch Esp Urol. 1969;XXII(4): 379.

31. II Curso de técnica quirúrgica de los trasplantes. Arch Esp Urol, 1969, XXII (4): 379 y Arch Esp Urol. 1970;XXIII(2): 213.

32. Congreso de Sociedad Internacional de Urología, Tokio, 1970. Arch Esp Urol. 1969;XXIII(3):314.

33. Gil-Vernet, JM. I Curso Internacional de Trasplante renal, Universidad de Barcelona, 1978. Actas Urol Esp. 1977;I (5).

34. Alférez C. Estadística Nacional de trasplantes renales. Congreso Nacional de Urol, León. Actas Asoc Esp Urol 1976;VIII(1):81-83.

35. Correas MA, Portillo JA, Gutiérrez JL, Monge JM, Roca A. Retrasplante renal. Estudio de 91 casos. Actas Urol Esp. 1993;27(7):430-433.

36. Leiva Ó, Díaz González R, Barrientos A, Fraile B, Borobia V. Estudio de la evolución del trasplante renal por medio del ultrasonido. Actas Urol Esp. 1978;2(6):301-304.

37. Franco Esteve A, Anaya Fernández F, Fernández de Caleya, Álvarez MA, Luño J, Martín Crespo L, et al. Complicaciones urológicas en 280 trasplantes renales, Actas Urol Esp. 1988;12(3):217-223.
38. Berenguer A, Lovaco F, Mayayo T, Escribano G, Llorente C, Orte L, et al. Complicaciones vasculares del trasplante renal. Actas Urol Esp. 1986;10(5):319-324.

39. Ballesteros Sampol JJ, Bosch García A, Guzmán Fernández A. Rara y asintomática complicación de la anastomosis pielo-piélica en un trasplante renal resuelta espontáneamente. Actas Urol Esp. 1983;7(5):387-388.

40. Berián Polo JM, Robles García JE, Zudaire Bergera AJ, Salvá A, Subirá ML, Erresti P, et al. Estudio retrospectivo de 101 trasplantes renales. Parte I: Resultados globales. Parte II: Complicaciones vasculares. Parte III: Complicaciones urinarias. Parte IV: Otras complicaciones quirúrgicas. Actas Urol Esp. 1985;9(6):481-500.

41. Beamud Gómez A, Estornel Moragues F, Martínez Verduch M, García Ibarra F, et al. Trasplante renal en niños. A propósito de 79 casos. VIII Reunión Nacional de Urotrasplante. Enero de 1987, Andorra.

42. Pertusa Peña C, Llarena R, Lecumberri D, Fernández del Busto E, et al. Relación entre trasplante renal y relación laboral. Arch Esp Urol. 1997;50(5):489-494.

43. Montañés Medina P, Sánchez Gómez E, Torrubia Romero FJ. Complicaciones quirúrgicas del trasplante renal, Tema monográfico LXVII Congreso Nacional de Urología, Actas Urol Esp. 1992.

44. Requena Tapia MJ, Ruiz Álvarez-Cienfuegos F, Saceda López,JL, Molina Sánchez J, et al. Complicaciones en 60 trasplantes renales. Actas Urol Esp. 1986;10(5):325-330.

45. Gutiérrez Calzada JL, Ramos Tito J, González Bonilla JA. Trasplante renal: Anomalias vasculares y procedimientos de revascularización renal. Actas Urol Esp. 1982;6(5):265-272.

46. González L, Núñez J, Torres C, Zuluaga A. Evolución de la lisozima urinaria en el trasplante renal. Actas Urol Esp. 1985;9(1):59-62.

47. Diz M, Anitúa, M. Riñón derecho y trasplantación renal. Arch Esp Urol. 1973;26(3):241-253.

48. Diz M, Anitúa M. Nuestra técnica de extracción renal en el cadáver para acortar el tiempo de isquemia caliente. Arch Esp Urol. 1973;26(6):577-599.

49. Gil-Vernet JM, Carretero P, Ballesteros JJ, Figuls J, Guerra C, Tinat J. Aspectos técnicos del trasplante renal. Extracción del riñón y homotrasplante. Reuniones Regionales de la AEU. 1976, № extra, XLI Congreso Nacional de Urología: 47-53.

50. Guerra C, Figuls F, Carretero P, Ballesteros JJ, Peyri E, Masramón $\mathrm{J}$, et al. Infección urinaria después del trasplante renal y otros cuadros infecciosos. Reuniones Regionales de la AEU. 1976, № extra, XLI Congreso Nacional de Urología: 55-63.

51. Ballesteros JJ, Carretero P, Figuls J, Benitez C, Lloveras J, Gelabert A, et al. Complicaciones quirúrgicas del trasplante renal. Reuniones Regionales de la AEU. 1976, № extra, XLI Congreso Nacional de Urología: 63-73.

52. Figuls J, Carretero P, Ballesteros JJ, Felipe F, Rotger F, Millones H, et al. Resultados de la anastomosis pielo-piélica en el trasplante renal", Reuniones Regionales de la AEU. 1976, № extra, XLI Congreso Nacional de Urología: 73-78.

53. Benitez C, Ballesteros JJ, Figuls J, Andreu J, Guerra C, Masramón J. Método de conservación del riñón aislado. Reuniones Regionales de la AEU. 1976, № extra, XLI Congreso Nacional de Urología: 79-82.

54. López A, Rosas S, Dorado J, Mallafré JM, Lloveras J, Andrés E, et al. Yatrogenia en el trasplante renal. Reuniones Regionales de la AEU. 1976, № extra, XLI Congreso Nacional de Urología: 83-94. 
55. Bonet Vic J, Guerra C, Carretero P, Figuls J, Ballesteros, JJ. Trasplante renal e hipertensión, Reuniones Regionales de la AEU. 1976, № extra, XLI Congreso Nacional de Urología: 295-301.

56. Berian JM, Purroy A, Arcas R, Subira ML, Martínez-Lage M. Consideraciones para la colaboración en el programa de trasplantes de riñón. Reuniones Regionales de la AEU. 1977;I(2):485-506.

57. Miranda B, Matesanz JA, Fernández Represa JA. Historia del trasplante renal en España, en "Estado actual del trasplante renal”. Clínicas Urológicas de la Complutense. 1999;(7):15-31.

58. Villavicencio H, Lauricia O, Sánchez Martín F, Caparros J Salvador J. Complicaciones urológicas en el trasplante renal. Nuestra experiencia en 200 casos. Actas Urol Esp. 1992;26(10):759-768.

59. Riera Canals L, Armora J, López Costea MA, Vigués F, Bordalba JR, Serón P et al. Trasplante renal en mayores de 50 años. Nuestra experiencia en 58 casos. Actas Urol Esp. 1992:26(1):44-50.

60. Osca García JM, Alonso Gorrea M, Pallardo L, Morera J, Ruiz-Cerdá JL, Guillén Navarro M, et al. Infección de herida operatoria en trasplantados renales. Actas Urol Esp. 1993;27(3):176-180.

61. Rodríguez García J, García Buitrón I, Chantada V, García Freire C, Picallo J, González Martín M, Trasplantectomía renal. Actas Urol Esp. 1992;26(1): 25-28.

62. Vivancos Garbayo S, Castro Díaz D, Hernández Marrero D, Concepción Masip T, Rodríguez Vera JM, Gutiérrez Hernández P, et al. Complicaciones quirúrgicas en 1.000 trasplantes renales, Actas Urol Esp. Supl. 2000;24(5):83. P-60.

63. Cimadevilla A, Lamas P, Marqués A, Ríos L. Complicaciones quirúrgicas en 90 trasplantes renales. IV Reunión Nacional de Urotrasplante, Granada, 1989.

64. Romero Tenorio M, Gutiérrez Somoscarrera JA, Ibáñez Suárez R, Solano D, Salazar S, Flores Giner J. Complica- ciones quirúrgicas tras 160 trasplantes renales. Experiencia. Comentarios. Arch Esp Urol. 1994;47(4):385390.

65. Server G, Pérez Albacete M, Salmerón G, Guardiola A, Gómez G, Martínez Pertusa P. Comunicación a la estadística nacional de los trasplantes del Hospital Virgen de la Arrixaca de Murcia. VIII Reunión Nacional de Urotrasplante. Enero de 1987, Andorra.

66. Muruamendiaraz V, Martínez Gómez FJ, Tamargo I, Martínez Benito JL, Alonso Saiz F. Estadística y resultados de los trasplantes en el Hospital Covadonga. VIII Reunión Nacional de Urotrasplante. Enero de 1987, Andorra.

67. Cavero Rebollo O, Urruchi P, Liedana JM, Roncalés A, Rioja C, Gutiérrez Colón JA, et al. Trasplante renal: resultados. Actas Urol Esp. 1992;16(9):695-699.

68. Mira A. Pérez-Llorca L, Pelluch A, Lobato J, Megías J, Romero P. Donación, extracción, implante. Factores condicionantes y casuística de nuestro Hospital. 1987-1988. IV Reunión Nacional de Urotrasplante, Granada, 1989.

69. Vicente Prados FJ, Martínez Morcillo A, Campos González MJ, Tallada M, Pedrajas G, Espejo E, et al. Nuevos marcadores biológicos en el diagnóstico del rechazo agudo y la disfunción inicial del injerto. Actas Urol Esp. 1999;23(5):8 2. C-47.

70. Gutiérrez del Pozo R, Ricart Brulles MJ, Bacque MC, Fernández Cruz L, Talbot-Wright $\mathrm{P}$, Carretero P. Trasplante renopancreático. Complicaciones urológicas. Actas Urol Esp. 1997;21(10):950-955.

Dr. M. Pérez Albacete

E-mail: Mariano.perez2@carm.es

(Trabajo recibido el 5 de junio de 2006) 